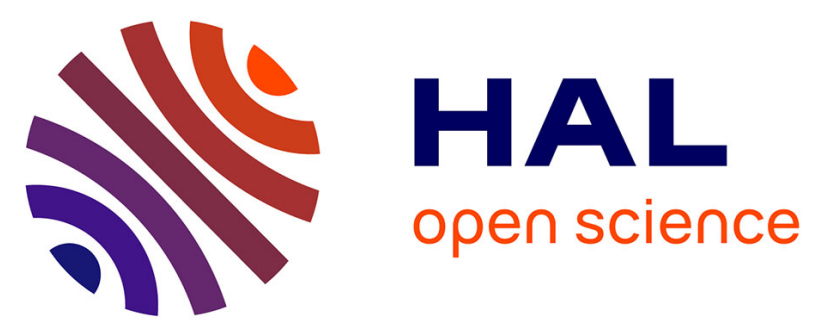

\title{
PolyRMPC: a New Matlab Toolbox to Robustify Predictive Controllers under Unstructured and Structured Uncertainties
}

V. Preda, Cristina Stoica, Pedro Rodriguez-Ayerbe, Didier Dumur

\section{- To cite this version:}

V. Preda, Cristina Stoica, Pedro Rodriguez-Ayerbe, Didier Dumur. PolyRMPC: a New Matlab Toolbox to Robustify Predictive Controllers under Unstructured and Structured Uncertainties. 2013 IEEE Conference on Computer Aided Control System Design (CACSD), Aug 2013, Hyderab, India. pp.328333, 10.1109/CACSD.2013.6663484 . hal-00826646

\section{HAL Id: hal-00826646 \\ https://hal-centralesupelec.archives-ouvertes.fr/hal-00826646}

Submitted on 16 Mar 2020

HAL is a multi-disciplinary open access archive for the deposit and dissemination of scientific research documents, whether they are published or not. The documents may come from teaching and research institutions in France or abroad, or from public or private research centers.
L'archive ouverte pluridisciplinaire HAL, est destinée au dépôt et à la diffusion de documents scientifiques de niveau recherche, publiés ou non, émanant des établissements d'enseignement et de recherche français ou étrangers, des laboratoires publics ou privés. 


\title{
PolyRMPC: a New Matlab Toolbox to Robustify Predictive Controllers under Unstructured and Structured Uncertainties
}

\author{
V. Preda, C. Stoica, P. Rodríguez-Ayerbe and D. Dumur
}

\begin{abstract}
This paper proposes a new Matlab toolbox to simplify the task of designing robustified Model Predictive Controllers under both unstructured and structured uncertainties. With a user-friendly design, the toolbox offers the possibility to develop an initial stabilizing predictive controller to handle both Single-Input Single-Output or multivariable systems. Using the Youla-Kučera parametrization, this initial control law can be further robustified under unstructured and polytopic uncertainties, while respecting time-domain specifications for disturbances rejection. Due to its visualization facilities, the performances of the developed controllers can be easily analyzed and compared in both time and frequency domains. Combining Object Oriented Programming with Matlab Graphical User Interface, this toolbox is built on top of a clear solid framework offering the possibility to extend and update the software design.
\end{abstract}

\section{INTRODUCTION}

This paper stands at a crossroad between two important branches of control strategies: predictive and robust control techniques. Both of these approaches have proven their value and are an ongoing source of interest for industrial and academic communities [1], [2], [3], [4], [5]. However designing such control strategies seems sometimes difficult to non-specialists. Matlab Robust Control Toolbox can be used to design robust controllers under different uncertainties. Matlab Model Predictive Control Toolbox offers the possibility to develop predictive controllers acting despite existing disturbances or noises, but no uncertainties are considered during the predictive controller synthesis. The main motivation of our work arises in order to overcome the difficulties in designing, tuning and analyzing off-line robustified predictive control techniques on different uncertain systems.

The purpose of this paper is to present a toolbox (called PolyRMPC) to be used for off-line robustification of model predictive controllers with respect to structured and unstructured uncertainties. In a first stage, the toolbox offers the possibility to design an initial stabilizing model predictive controller for a nominal model of the real system. The next stage consists in robustifying this initial controller under unstructured additive and/or multiplicative uncertainties [6], [7]. This robustification problem can be formulated as a convex optimization problem of a Youla parameter (also

V. Preda is with Politehnica University of Bucharest, Faculty of Automatic Control and Computer Science, 313 Splaiul Independentei, Bucharest, 060032, Romania (e-mail: mail@valentinpreda.ro). C. Stoica, P. Rodríguez-Ayerbe and D. Dumur are with SUPELEC Systems Sciences (E3S) - Automatic Control Department, 3 rue Joliot Curie, F91192, Gif-sur-Yvette cedex, France (e-mail: \{cristina.stoica; pedro.rodriguez; didier.dumur\}@supelec.fr). called $Q$ parameter) that can be efficiently solved using existing LMI (Linear Matrix Inequalities) solvers. Time domain specifications for disturbances rejection can be added to the considered LMI problem [7]. A previous version of such a toolbox [8] already handled these specification demands, but the data entry appeared time-demanding and sometimes unappropriate for users. Moreover this structure proved to be inappropriate for addressing the robust stability under polytopic uncertainties. In order to solve these problems, a new toolbox based on Object Oriented design with a new user-friendly interface has been developed. Systems can be imported from a file or from the workspace, as state-space representations or transfer functions. Uncertain state-space models can be used, which allowed adding a new module that guarantees robust stability under polytopic uncertainties. This is the main theoretical novelty of our toolbox. Synthesizing a robustified controller to deal with polytopic uncertainties is a hard task, because of the nonconvexity of the optimization problem, which is a BMI (Bilinear Matrix Inequality). An efficient algorithm to find a sub-optimal solution is implemented instead of using existing BMI solvers that may be time-demanding.

The PolyRMPC toolbox allows expert or non-specialist users to easily solve the problems described above. The software offers a clean and intuitive interface to help users easily design and compare the performances of robustified predictive controllers. A module for order reduction of the obtained controller is also proposed.

This paper is organized as follows. Section II reminds the theoretical background which is the basis of the toolbox development. Section III describes the proposed toolbox architecture, offering details on each window. An application on the model of a Quanser helicopter is proposed in Section IV in order to highlight the toolbox performance. Finally, concluding remarks and perspectives are drawn in Section V.

\section{Problem Formulation}

This section provides the necessary theoretical aspects on which the toolbox is based. First, an initial stabilizing predictive controller is developed and, second, it is robustified via the Youla-Kučera parametrization with respect to unstructured and structured uncertainties, while respecting time-domain constraints for disturbances rejection.

\section{A. Initial Stabilizing MPC}

Consider the nominal linear discrete-time invariant system:

$$
\left\{\begin{array}{l}
x(k+1)=A x(k)+B u(k) \\
y(k)=C x(k)
\end{array} \Leftrightarrow(A, B, C, \mathbf{0})\right.
$$


with $x \in \mathbb{R}^{n}$ the state vector, $u \in \mathbb{R}^{m}$ the control input vector, $y \in \mathbb{R}^{p}$ the output vector, and the matrices $A, B, C$ of appropriate dimensions, with $(A, B)$ controllable, $(A, C)$ observable. An integral action $u(k)=u(k-1)+\Delta u(k)$ is added to the state equation in order to cancel the steadystate errors leading to an extended state-space representation $\left(A_{e}, B_{e}, C_{e}, \mathbf{0}\right)$, with $x_{e}(k)=\left[\begin{array}{ll}x^{T}(k) & u^{T}(k-1)\end{array}\right]^{T}$. The control signal (Fig. 1):

$$
\Delta u(k)=y_{F r}(k)-L x_{e}(k)=y_{F r}(k)-L_{1} x(k)-L_{2} u(k-1),
$$

defined in a general way by a feedback vector $L=$ $\left[\begin{array}{ll}L_{1} & L_{2}\end{array}\right] \in \mathbb{R}^{m \times(n+m)}$ and a filtered set-point $y_{F r}$, is the result of the minimization of the cost function:

$$
J=\sum_{i=N_{1}}^{N_{2}}\left\|\hat{y}(k+i)-y_{r}(k+i)\right\|_{\tilde{Q}}^{2}+\sum_{i=0}^{N_{u}-1}\|\Delta u(k+i)\|_{\tilde{R}}^{2}
$$

with $N_{1}, N_{2}$ the output prediction horizons, $N_{u}$ the control prediction horizon, $\tilde{Q}, \tilde{R}$ positive definite diagonal weighting matrices, $\hat{y}$ the predicted output vector, $y_{r}$ the set-point vector and $\Delta u(k+i)=0, i \geq N_{u}$. If the state-vector is unknown, it can be estimated by means of an observer. For simplicity, the following complete observer is proposed here:

$$
\hat{x}_{e}(k+1)=A_{e} \hat{x}_{e}(k)+B_{e} \Delta u(k)+K\left[y(k)-C_{e} \hat{x}_{e}(k)\right]
$$

with the observer gain $K$ designed by pole placement.

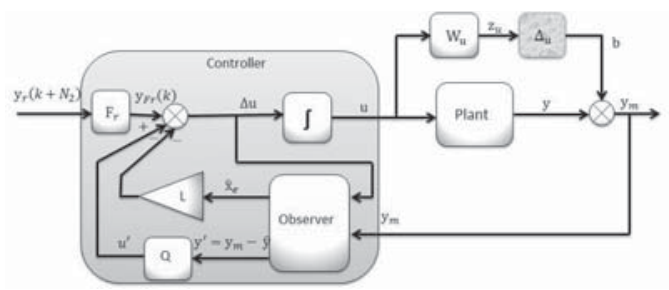

Fig. 1. Robustified MPC w.r.t. additive uncertainties via the $Q$ parameter

\section{B. Robust Stability under Unstructured Uncertainties}

Starting from the initial stabilizing controller (2), a stable Youla-Kučera parameter $Q$ [4] can be used to construct the set of all stabilizing controllers for system (1). If inserted in a particular way (see the modified controller paradigm [9]), a stable $Q \in \mathbb{R} H_{\infty}$ parameter allows to improve the robustness (w.r.t. different sensitivity functions) of the controlled system, without changing the nominal performances of the Input-Output (I/O) behavior [10], [7]. A suboptimal solution [6] of the $Q$ parameter is implemented in this toolbox, consisting into a FIR (Finite Impulse Response) filter of degree $n_{Q}$, in the state-space representation $\left(A_{Q}, B_{Q}, C_{Q}, D_{Q}\right)$. As in [6], a fixed pair $\left(A_{Q}, B_{Q}\right)$ is chosen, while the variable pair $\left(C_{Q}, D_{Q}\right)$ is the result of an off-line optimization problem. This leads to the following control law $\Delta u(k)=y_{F r}(k)-L x_{e}(k)-u^{\prime}(k)$ (Fig. 1).

Applying the small gain theorem [4] to the system subject to additive unstructured uncertainties $\Delta_{u}$ (Fig. 1) guarantees that if the condition $\left\|T_{z_{u} b}\right\|_{\infty}<\gamma$ on the transfer from $b$ to $z_{u}$ is satisfied, then the controlled system is stable for all additive unstructured uncertainties with $\left\|\Delta_{u}\right\|_{\infty} \leq \gamma^{-1}$. This leads to find a stabilizing controller (i.e. a stable $Q$ parameter) that solves the following optimization problem:

$$
\min _{Q}\left\|T_{z_{u} b}\right\|_{\infty}=\min _{Q}\left\|W_{u} T_{u b}\right\|_{\infty}
$$

where the $W_{u}$ weight can be set to satisfy the robustness specifications in the desired frequency range.

This minimization problem can be reformulated using the bounded real lemma [5] into a LMI, denoted here $L M I_{0}$. The goal is to find $X_{1}=X_{1}^{T} \succ 0$ (corresponding to the Lyapunov variable), the $Q$ parameter (i.e. $C_{Q}$ and $D_{Q}$ matrices) and $\gamma$ in the following optimization problem:

$$
\begin{aligned}
& \min _{Q, X_{1}, \gamma} \gamma \\
& \text { s.t. } L M I_{0}
\end{aligned}
$$

This LMI formulation is beyond the scope of this paper and it is omitted here (more details are given in [7]).

In a similar way, robust stability under multiplicative unstructured uncertainties $\Delta_{y}$ (with $\left\|\Delta_{y}\right\|_{\infty} \leq \widetilde{\gamma}^{-1}$ ) can be addressed. In this case, the aim is to find a stabilizing controller that solves the following optimization problem:

$$
\min _{Q}\left\|T_{z_{y} b}\right\|_{\infty}=\min _{Q}\left\|W_{y} T_{y b}\right\|_{\infty}
$$

with the weight $W_{y}$. This minimization problem can also be reformulated using the bounded real lemma into a LMI. The goal is to find $\widetilde{X}_{1}=\widetilde{X}_{1}^{T} \succ 0$, the $Q$ parameter and $\widetilde{\gamma}$ in:

$$
\begin{aligned}
& \min _{Q, \widetilde{X}_{1}, \widetilde{\gamma}} \widetilde{\gamma} \\
& \text { s.t. } L M I_{m}
\end{aligned}
$$

with $L M I_{m}$ obtained from expression $L M I_{0}$ [7] after replacing $X_{1}, \gamma, W_{u}$, and $T_{z_{u} b}$ with $\widetilde{X}_{1}, \widetilde{\gamma}, W_{y}$, and $T_{z_{y} b}$, resp.

\section{Robust Stability under Polytopic Uncertainties}

This subsection deals with robust stability under polytopic uncertainties around a nominal model denoted $\left(A_{0}, B_{0}, C_{0}, 0\right)$, i.e. the matrices of system (1) belong to the convex hull Co $\left\{\left[\begin{array}{lll}A_{1} & B_{1} & C_{1}\end{array}\right],\left[\begin{array}{lll}A_{2} & B_{2} & C_{2}\end{array}\right], \ldots,\left[\begin{array}{lll}A_{l} & B_{l} & C_{l}\end{array}\right]\right\}$. For the given uncertain system, the following equality holds $\left[\begin{array}{lll}A & B & C\end{array}\right]=\sum_{i=1}^{l} \lambda_{i}\left[\begin{array}{lll}A_{i} & B_{i} & C_{i}\end{array}\right]$, with $\sum_{i=1}^{l} \lambda_{i}=1$, for $\lambda_{i} \geq 0, l$ being the number of vertices. To guarantee the stability across the polytopic domain, the expression (9) [11] has to be satisfied for each vertex $i$ of the polytope:

$$
\left[\begin{array}{cc}
X_{2, i} & A_{c l, i}(Q)^{T} G^{T} \\
G A_{c l, i}(Q) & G+G^{T}-X_{2, i}
\end{array}\right] \succ 0, \quad i=\overline{1, l}
$$

with the decison variables $X_{2, i}=X_{2, i}^{T} \succ 0$ and the $Q$ parameter enclosed in the closed-loop matrices $A_{c l, i}(Q)$.

The goal of the optimization procedure is to robustify under additive uncertainties while also guaranteeing stability across the polytopic domain. This global robustness problem can be stated as the following mathematical expression:

$$
\begin{aligned}
& \min _{Q, X_{1}, \gamma, X_{2, i}, G} \gamma \\
& \text { s.t. } L M I_{0}, B M I_{i}
\end{aligned}
$$


where $B M I_{i}$ denotes the Biliniar Matrix Inequality (9), with $i=\overline{1, l}$, in order to test stability across the polytope. BMI problems are non-convex and hence difficult to solve. Existing (local) solvers may be time-consuming and are not always available for free. In order to overcome these inconveniences and to keep our toolbox 'autonomous', an improved tractable sub-optimal solution [12] of (10) is implemented into the toolbox. The idea behind this algorithm is that the robustification procedure w.r.t. additive uncertainties can lead to a controller that offers stability only across a part of the polytope, while the robustification procedure w.r.t. multiplicative uncertainties can enlarge this polytopic stability domain (but at a cost to the performance in the frequency domain of the nominal model). The goal is to find a controller with a stability domain that fully covers the given polytope while also being robust to additive uncertainties and thus having a small $H_{\infty}$ norm for the $T_{u b}$ transfer (Fig. 1).

\section{Algorithm}

1) Initialization: Find an initial controller with a large stability domain that covers the whole polytope by solving the robustification procedure for both additive and multiplicative uncertainties:

$$
\begin{aligned}
& \min _{Q, X_{1}, \widetilde{X}_{1}, \gamma, \widetilde{\gamma}} c \gamma+\widetilde{c \gamma} \\
& \text { s.t. } L M I_{0}, L M I_{m}
\end{aligned}
$$

with the tuning parameters $c, \widetilde{c}, W_{u}, W_{y}$ and $n_{Q}$. A Youla parameter $Q^{*}$ is found.

2) Find the Lyapunov function: With the newly found $Q^{*}$ parameter, the BMI expressions (9) are converted to LMIs by eliminating the Youla parameter from the decision variables leading to $\left[\begin{array}{cc}X_{2, i} & A_{c l, i}\left(Q^{*}\right)^{T} G^{T} \\ G A_{c l, i}\left(Q^{*}\right) & G+G^{T}-X_{2, i}\end{array}\right] \succ 0$, for $i=\overline{1, l}$. A feasible solution $\left(X_{2, i}^{*}, G^{*}\right)$ of these LMIs is searched.

3) Find the Youla parameter: The values $X_{2, i}^{*}$ and $G^{*}$ obtained from the previous step can be used to replace the decision variables $X_{2, i}$ and $G$ in the BMIs (9) leading to the LMIs given below $\left[\begin{array}{cc}X_{2, i}^{*} & A_{c l, i}(Q)^{T} G^{* T} \\ G^{*} A_{c l, i}(Q) & G^{*}+G^{* T}-X_{2, i}^{*}\end{array}\right] \succ 0$, for $i=\overline{1, l}$. This new set of LMIs is added to the previous minimization problem with respect to additive uncertainties (6), being a sub-optimal solution of the problem (10).

This way, the solution found is certain to be stable for all the values inside the polytopic domain. By iterating the last two steps, the solution converges to a local minimum. The algorithm stops once the solution cannot be improved upon or when the changes made to the solution after a given number of iterations are insignificant.

Questions about the feasibility of this algorithm can arise. In fact, the most difficult step is to find a set of parameters $c$, $\widetilde{c}, W_{u}, W_{y}$ and $n_{Q}$ that guarantees the stability on a domain which includes the considered polytopic domain. To facilitate this task, a stability check is implemented in the toolbox.

\section{Time-domain Specifications for Disturbances Rejection}

Nominal performance specifications can be added in terms of additional LMIs to the optimization problems considered in Section II.B and II.C. These nominal performance specifications are actually time-domain templates [7] on disturbances rejection, used to reduce the impact of the disturbances on the system outputs. These specifications can be written as a LMI (denoted $L M I_{t}$ ) [7] added to the previously built optimization problems (6), (8) or (10), leading to the new global optimization problem:

$$
\begin{aligned}
& \min _{Q, X_{1}, \gamma, X_{2, i}, G} \gamma, \quad \forall i=\overline{1, l} \\
& \text { s.t. } L M I_{0}, B M I_{i}, L M I_{t}
\end{aligned}
$$

Because the notion of nominal performance and robust stability are complementary, there is a trade-off (handled by the Youla parameter) between them. If the time-domain templates are too restrictive, the optimization problem can become infeasible and the templates need to be relaxed.

\section{TOOLBOX DESCRIPTION}

Based on the theoretical background presented in Section II, this section focuses on the main features of the developed toolbox, offering an analysis of the main windows.

\section{A. Main Features and Software Architecture}

With a user-friendly design, the PolyRMPC toolbox relies on a modular software architecture based on objectoriented design techniques, allowing easy code maintenance and debugging while also providing greater flexibility to extend the codebase with new features. Examples of the objects used to model different subsystems throughout the controller design or analysis procedures include: MPController (keeps all relevant information about a controller), Simulation Options (stores the various set-points and disturbances acting on different channels), Optimization Settings (stores information needed to perform the various robustification procedures), Disturbance Rejection Template (holds the necessary information about the limits imposed on the disturbance response). The toolbox handles both multivariable and SISO (Single-Input Single-Output) discrete-time systems, introduced either as transfer functions or state-space representations. An option allowing to import a system or a model from an existing file / workspace has been added.

Features are also included to aid in the design of the initial MPC controller. It is thus easy to automatically generate an appropriate observer for the control system or to quickly set the appropriate weights for the MPC objective function.

The possibility of using uncertain state-space representations allows handling robustness of systems with parametric uncertainties. Model uncertainties are incorporated using the uss (uncertain state-space) object provided in the MATLAB Robust Control Toolbox. The stability across the polytopic domain for a given controller can be asserted.

Many features are also included in the analysis part: time-domain settings and analysis, frequency analysis of the developed controllers. A new feature concerns exporting 
controllers in a reduced form. A Hankel singular values analysis is proposed in order to help the user to choose the states to be eliminated. Model-order reduction can also be performed on the Youla parameter and the consequences of this approximation can be analyzed. In addition, the PolyRMPC toolbox can save or load existing projects, which proves to be an useful option.

\section{B. Overview of PolyRMPC Toolbox}

Due to space limitations, Fig. 2 regroups the main windows of PolyRMPC, described as follows.

Main Window (Fig. 2.1): Displayed when the application starts, it is composed of four panels: Plant, Plant Models, Controllers and Analysis. From here it is possible to import a plant (Fig. 2.2), to choose nominal models (Fig. 2.2) and to plot their characteristics (Fig. 2.3), to create, modify or delete controllers (Fig. 2.4), and to perform analysis in the time domain (Fig. 2.9, using a possible uncertain plant model) or in the frequency domain (Fig. 2.10). The File Menu allows to open a new project, an existing project and to save the project in progress. The MPC Menu allows to import a plant, a model or a previously created controller and to export a controller (Fig. 2.11).

Initial MPC Settings (Fig. 2.4): This window can be selected from the panels Controllers of the Main Window (Fig. 2.1). In a first stage, the parameters used to build an initial model predictive controller can be easily set or edited from this window. The user can select an appropriate plant model, choose the appropriate prediction horizons or control the dynamics of the observer using pole placement. Tracking error and control effort used in the controller design can be weighted using scalars, vectors or matrices to emphasize the importance of particular outputs or control signals. In a second stage, this initial predictive controller can be robustified by clicking on the Settings button.

Robustification Settings (Fig. 2.5): This window is used to adjust the parameters for the robustification under additive or multiplicative uncertainties and structured (polytopic) uncertainties: the degree of the Youla parameter, the transfer to be minimized (the optimization type), the frequency (input or output) weights as first or second order discrete-time filters (Fig. 2.6), or the time domain specifications (Fig. 2.7). The button Optimize starts the LMI optimization procedure. Advanced LMI optimization settings (e.g. relative accuracy, maximal number of iterations, feasibility radius, number of iterations to test convergence, trace execution of optimization in Fig. 2.8) can be considered by an experimented user. The same window is used to start the polytopic optimization procedure via the Polytopic Optimization button, to evaluate the stability across the polytopic domain (Check Polytope Stability button) or to perform a model order reduction on the computed Youla parameter (Youla Reduction button).

Time Domain Template Specification Window (Fig. 2.7): For each I/O channel, this window allows to choose the time-domain templates adjusting the appropriate sliders. The designed templates can be saved via the Set template button.
Time-domain Analysis (Fig. 2.9): Providing a method to easily compare the performances in time-domain of multiple controllers was clear objective from the beginning and it is accomplished via this window. For each I/O channel, individual set-points and input disturbances can be adjusted using the appropriate sliders. These changes are then reflected in the closed-loop system dynamics plots in the same window. Visualization of the time-domain specifications can also be enabled by selecting the checkbox labeled Show Templates, visible only if the current controller was optimized with timedomain specifications.

Frequency-domain Analysis (Fig. 2.10): This window allows to study the singular values for multivariable systems or the Bode diagram for SISO systems of different transfers for the selected controllers: the $T_{u b}$ transfer (Fig. 1), the direct or the complementary sensitivity functions.

Export Window - Model Order Reduction (Fig. 2.11): This window accomplishes the task of performing a model order reduction on the selected controller and exporting it as a workspace variable. The singular values of the selected controller and the reduced order controller can be compared.

\section{Application to a QuAnser Helicopter}

The Quanser 2-DOF Helicopter [13] consists of a helicopter model mounted on a fixed base. The pitch and the yaw propellers (driven by DC motors) are used to control the pitch and yaw angles of the model, resp. These angles are measured using two high resolution encoders. Different models exist in the literature [13], [14]. In order to focus on the control laws obtained with PolyRMPC, a simplified model [13] is further used. With the state vector composed by the pitch and yaw angles and related velocities, respectively, the corresponding continuous time state-space matrices are:

$$
\begin{aligned}
A & =\left[\begin{array}{cccc}
0 & 0 & 1 & 0 \\
0 & 0 & 0 & 1 \\
0 & 0 & -\frac{B_{p}}{J_{p}+m l^{2}} & 0 \\
0 & 0 & 0 & -\frac{B_{y}}{J_{y}+m l^{2}}
\end{array}\right], B=\left[\begin{array}{cc}
0 & 0 \\
0 & 0 \\
\frac{K_{p p}}{J_{p}+m l^{2}} & \frac{K_{p y}}{J_{p}+m l^{2}} \\
\frac{K_{y p}}{J_{y}+m l^{2}} & \frac{K_{y y}}{J_{y}+m l^{2}}
\end{array}\right], \\
C & =\left[\begin{array}{llll}
1 & 0 & 0 & 0 \\
0 & 1 & 0 & 0
\end{array}\right]
\end{aligned}
$$

where the $p$ index stands for pitch, the $y$ index stands for yaw, $J_{p}=0.0384 \mathrm{~kg} \mathrm{~m}^{2}$ and $J_{y}=0.0432 \mathrm{~kg} \mathrm{~m}^{2}$ are the inertia moments, $m=1.38 \mathrm{~kg}$ is the mass, $l=0.186 \mathrm{~m}$ is the center of mass length along the helicopter body from pitch axis, $K_{p p}=0.204 \mathrm{Nm} / \mathrm{V}, K_{p y}=0.0068 \mathrm{Nm} / \mathrm{V}, K_{y p}=$ $0.0219 \mathrm{Nm} / \mathrm{V}, K_{y y}=0.072 \mathrm{Nm} / \mathrm{V}$ are thrust torque constants, and the uncertain parameters $B_{p} \in[0.3,0.9] \mathrm{N} / \mathrm{V}$ and $B_{y} \in$ $[0.2,0.4] \mathrm{N} / \mathrm{V}$ are equivalent viscous damping factors.

\section{A. Robustification using PolyRMPC}

In the following, a step by step design of a robustified controller via the toolbox PolyRMPC is exemplified.

1) Model Import: Before any controller can be built, it is necessary to import a plant (eventually an uncertain system) and a nominal model. From the main window, clicking the Import button from the Plant Models panel opens up the Model Import window. The uncertain plant (13) will be used for time-domain analysis and polytopic optimization. 


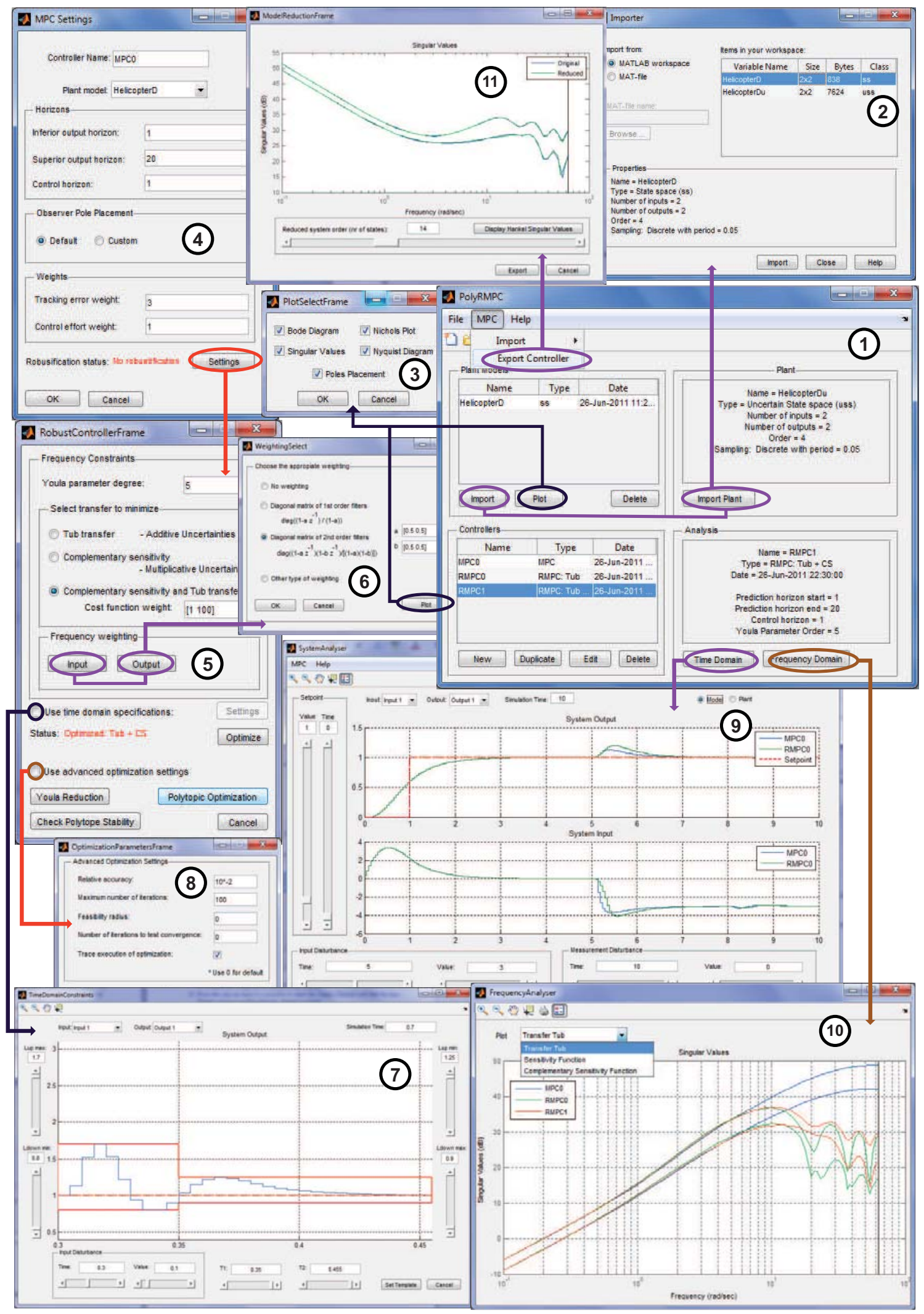

Fig. 2. PolyRMPC Overview: 1. Main Window, 2. Import Window, 3. Model Plot Window, 4. Initial MPC Settings Window, 5. Robust Controller Setting Window, 6. Weighting Select Window, 7. Time Domain Template Specification Window, 8. Advanced LMI Optimization Setting Window, 9. Time Domain Analysis Window, 10. Frequency Domain Analysis Window, 11. Export Window - Model Order Reduction. 
Only the state matrix $A$ contains the variable parameters. Thus only the $A$ matrix belongs to a polytope defined by four vertices corresponding to the extremes of the variation intervals for the $B_{p}$ and $B_{y}$ parameters: $A \in$ $\operatorname{Co}\left\{A_{1}, A_{2}, A_{3}, A_{4}\right\}$. Next, a first order discrete-time model with 2 inputs, 2 outputs and 4 states, sampled with a period of $0.05 \mathrm{~s}$, with the nominal values $B_{p_{0}}=0.8 \mathrm{~N} / \mathrm{V}$ and $B_{y_{0}}=$ $0.3 \mathrm{~N} / \mathrm{V}$, is chosen as the plant model (Fig. 2.2).

2) Initial MPC: Once the plant and its nominal model have been imported, they are displayed in the appropriate panels in the main window (Fig. 2.1). It is now possible to build an initial MPC controller (called MPC0) based on the nominal system via the New button in the Controllers panel, with the tuning parameters $N_{1}=1, N_{2}=20, N_{u}=1$, $\tilde{Q}=3 I_{2 N_{2}}, \tilde{R}=I_{2}$. Automatic pole placement is used for the observer. The time domain simulation (Fig. 2.9) was done using the nominal plant model, with a set-point of 1 rad for the pitch angle (output 1) and a setpoint of 0 to the yaw angle (output 2), step input disturbances with amplitude 3 and 8, at time 5 and 8, on input channel 1 and 2, resp.

3) Robustification to Additive Unstructured Uncertainties: Although the initial controller fulfills the desired nominal performances, it shows $\left\|T_{u b}\right\|_{\infty}=48.5 \mathrm{~dB}$ (Fig. 2.10). To improve the robustness, the initial MPC0 controller is duplicated (and renamed RMPC0) along with all the settings used to build it via Duplicate button in the Controllers panel.

The parameters for the robustification procedure are set: a FIR Youla parameter of order 5; a $T_{u b}$ transfer minimizing the objective for the robustification procedure w.r.t. additive unstructured uncertainties; a first order high-pass filter $W_{u}$ (set from the Frequency Weighting window). Then, clicking the Optimize button starts the robustification procedure.

To compare the performances in the frequency-domain for the two controllers MPC0 and RMPC0, it is necessary to first select them from the Controllers subpanel in the Main Window (Fig. 2.1) and then clicking on the Frequency Domain button. The Frequency Domain Analysis window (Fig. 2.10) is then revealed showing the singular values of the $T_{u b}$ transfer for the two controllers (Fig. 2.10). Robustness to additive uncertainties is significantly increased for the RMPC0 controller with $\left\|T_{u b}\right\|_{\infty}=36.8 \mathrm{~dB}$. Similarly, the time-domain performances can be studied by clicking the Time Domain button in Fig. 2.1. This action opens up the Time Domain Analysis window (Fig. 2.9) showing the input response of both controllers for the selected channel. The two controllers have the same response until a disturbance appears on the input channel (shown in Fig. 2.9). RMPC0 displays a higher peak response in the time-domain to the incoming disturbance.

4) Robustification to Polytopic Uncertainties: Let us address now the robust stability under the considered uncertain polytopic domain. The controller RMPC0 does not stabilise the considered polytopic domain. To begin the polytopic robustification procedure it is necessary to find an initial stabilizing solution (11). The goal is to find a controller that has similar performances as RMPC0 in the frequency domain, but it is also stable across the considered polytope.
First, a copy of RMPC0 called RMPC1 is made. Second, in order to modify RMPC1, the option Complementary sensitivity and $T_{u b}$ transfer from the Robustification Settings window (Fig. 2.5) is selected and the associated cost function weight $c=\left[\begin{array}{ll}c & \tilde{c}\end{array}\right]$ (see (11)) is adjusted until a stable solution across the entire polytopic domain is found. In this case a cost function of $c=\left[\begin{array}{ll}1 & 100\end{array}\right]$ is a good choice.

Next, the Polytopic Optimization button starts the optimization procedure using the default optimization parameters. Local convergence is reached within 7 iterations and the optimization procedure terminates leading to a $H_{\infty}$ of $38.6 \mathrm{~dB}$ for RMPC1 (Fig. 2.10). In the frequency domain, the trade-off between polytopic stability (RMPC1) and robustness to unstructured additive uncertainties (RMPC0) is made visible using the Frequency Domain Analysis window. In Fig. 2.10, the singular values of the $T_{u b}$ transfer for the closed-loop system using the RMPC0 and RMPC1 show high degree of similarity in high frequency range, with a relatively small increase in the $H_{\infty}$ norm obtained with RMPC1. But this slight compromise guarantees the polytopic stability.

\section{CONCLUSION}

This paper presented a new Matlab toolbox used for robustification of unconstrained Model Predictive controllers under both structured and unstructured uncertainties, while respecting time-domain specifications for disturbances rejection. A theoretical contribution is to consider the robustification method with respect to polytopic uncertainties. Another novel element of our toolbox is given by the Object Oriented architecture that increases the software reliability.

\section{REFERENCES}

[1] E. F. Camacho and C. Bordons, Model predictive control. London: Springer-Verlag, 2004.

[2] S. J. Qin and T. Badgwell, "A survey of industrial model predictive control technology," Control Engineering Practice, vol. 11, 2003.

[3] Z. Wan and M. V. Kothare, "Robust output feedback model predictive control using off-line linear matrix inequalities,” in Proc. of the ACC, Arlington, USA, 2001.

[4] K. Zhou, J. C. Doyle, and K. Glover, Robust and optimal control. Prentice Hall, 1996.

[5] S. Boyd, L. El Ghaoui, E. Feron, and V. Balakrishnan, Linear matrix inequalities in system and control theory. SIAM Publications, 1994.

[6] C. W. Scherer, "An efficient solution to multi-objective control problem with LMI objectives,” Systems and Control Letters, 2000.

[7] C. Stoica, P. Rodríguez-Ayerbe, and D. Dumur, "Off-line improvement of multivariable model predictive control robustness," in Proc. of the 46th IEEE CDC, New Orleans, L.A., USA, 2007.

[8] C. Stoica, P. Rodríguez-Ayerbe and D. Dumur, "MIMOptMPC: a MATLAB toolbox for off-line robustification of multivariable MPC," in Proc. of IEEE MSC, San Antonio, Texas, USA, 2008.

[9] S. Boyd and C. Barratt, Linear controller design. Limits of performance. Prentice Hall, 1991.

[10] J. A. Rossiter, Model based predictive control. A practical approach. CRC Press LLC, 2003.

[11] M. C. de Oliveira, J. Bernussou, and J. C. Geromel, "A new discretetime robust stability condition,” Systems and Control Letters, 1999.

[12] C. Stoica, P. Rodríguez-Ayerbe and D. Dumur and S. Tebbani, "Towards tractable off-line robustified controllers for uncertain systems," in Proc. of 10th IEEE ICARV, Hanoi, Vietnam, 2008.

[13] K. M. Passino, Quanser 2-DOF Helicopter Reference Manual. available at http://www2.ece.ohio-state.edu/passino/Quanser2DOFheli.pdf.

[14] B. Cazzolato, 2DOF Quanser Helicopter Tutorial and Lab. available at http://personal.mecheng.adelaide.edu.au/ben jamin.cazzolato/Quanser/Automatic_Control_II_Heli_V9.pdf, 2006. 